\title{
AUTOMATIC LUNG NODULE DETECTION BASED ON STATISTICAL REGION MERGING AND SUPPORT VECTOR MACHINES
}

\author{
Elaheh Aghabalaei Khordehchi, Ahmad Ayatollahi ${ }^{\circledR}$, MOHAMmad ReZa DaliRi \\ Iran University of Science and Technology, Electrical Engineering Department, Tehran, Iran \\ e-mail: elaheh.aghabalaiee@gmail.com, ayatollahi@iust.ac.ir, daliri@iust.ac.ir \\ (Received December 14, 2016; revised February 5, 2016; accepted March 21, 2017)
}

\begin{abstract}
Lung cancer is one of the most common diseases in the world that can be treated if the lung nodules are detected in their early stages of growth. This study develops a new framework for computer-aided detection of pulmonary nodules thorough a fully-automatic analysis of Computed Tomography (CT) images. In the present work, the multi-layer CT data is fed into a pre-processing step that exploits an adaptive diffusionbased smoothing algorithm in which the parameters are automatically tuned using an adaptation technique. After multiple levels of morphological filtering, the Regions of Interest (ROIs) are extracted from the smoothed images. The Statistical Region Merging (SRM) algorithm is applied to the ROIs in order to segment each layer of the CT data. Extracted segments in consecutive layers are then analyzed in such a way that if they intersect at more than a predefined number of pixels, they are labeled with a similar index. The boundaries of the segments in adjacent layers which have the same indices are then connected together to form three-dimensional objects as the nodule candidates. After extracting four spectral, one morphological, and one textural feature from all candidates, they are finally classified into nodules and non-nodules using the Support Vector Machine (SVM) classifier. The proposed framework has been applied to two sets of lung $\mathrm{CT}$ images and its performance has been compared to that of nine other competing state-of-the-art methods. The considerable efficiency of the proposed approach has been proved quantitatively and validated by clinical experts as well.
\end{abstract}

Keywords: CT X-ray images, morphological filtering, nodule detection, nonlinear partial differential quations, statistical region merging, support vector machine.

\section{INTRODUCTION}

Pulmonary nodules are defined as abnormal radiographic opacities which are located in the lung area and surrounded by normal tissues (Gould et al., 2007; Alpert et al., 2015; Kim et al., 2003). Such growths (which may be solitary or multiple) are often less than $30 \mathrm{~mm}$ in diameter (Gould et al., 2007). Computeraided detection of the pulmonary nodules can play an important role in diagnosis and therapy of lung cancer which itself is one of the most common types of cancer.

There are numerous non-invasive imaging modalities that have been developed for medical diagnosis applications. Among them, one of the most widelyused procedures is Computed Tomography (CT) in which several X-ray images are taken from different angles in order to provide a detailed representation of the tissues and organs of the body (Herman, 2009). The CT imaging leads to cross-sectional views of the scanned object and hence produces a multi-layer data which often conveys more information about the inside of the object. Due to its high spatial resolution and relatively low cost, CT technology appears to be the most common diagnostic modality for producing medical images (El-Baz et al., 2013, Hoffman et al., 2004).

In the lung imaging applications, $\mathrm{CT}$ is a common choice. More specifically, computer-aided analysis of CT images is very beneficial in automatic detection of lung nodules. Most of the methods dealing with detection of nodules in the CT images are based on segmentation of the CT data. Among these methods, the threshold-based approaches seem to be in majority. Some examples of the threshold-based segmentation approaches are reviewed in the following paragraphs.

In (Hu et al., 2004), opening and closing morphological operations were used in order to smooth the boundaries and then gray-level thresholding were applied for segmenting the lung CT images. Authors in (Yim et al., 2005) utilized threshold-based region growing as well as connected component analysis for CT image segmentation. In their approach, two levels of region growing were used. In the first level, an 
inverse seeded region growing was applied while a three-dimensional region growing was exploited in the second level. Their work was successful in accurately determining the lung area even in cases in which the lungs contained abnormal objects such as tumors or pulmonary emboli. However, it was not able to detect such objects, individually (Yim et al., 2005). In (Gao et al., 2007), a threshold-based segmentation approach was proposed in which the thresholds used for removing the lung artery and separating the left and right lungs were set by an optimization technique. Authors in (Hassanpour et al., 2011) proposed a segmentation method based on an innovative concept named as Pixon. Their approach followed a thresholdbased region growing methodology. After the segmentation process, fuzzy clustering were used in order to merge those segments which were not large enough. They also applied wavelet thresholding for denoising the input images (Hassanpour et al., 2011). Benefiting from histogram analysis of the CT images in order to tuning the threshold and then through a connected component labeling technique, authors in (Wei and $\mathrm{Li}, 2014)$ developed a segmentation method used for lung nodule detection.

In (Hasanabadi et al., 2014), lung nodules were detected by thresholding and then template matching. Authors in (John and Mini, 2016) proposed a multilevel (three levels) thresholding technique for detection of the pulmonary nodules. Thresholding technique was also used in (Akram et al., 2016) for segmentation of the image. Using geometric and intensity based features which were statistically obtained from the data, the support vector machine classifier was then trained for distinguishing the lung nodules from the other normal tissues.

Although the above-mentioned methods are often able to properly segment the CT images and then extract the nodules, they are not robust enough to cover a wide range of data. To be clearer, some normal pulmonary structures such as arteries, veins and bronchial tubes may have similar density (measured in Hounsfield units) to that of the nodules. Therefore, threshold-based segmentation may lack adequate accuracy especially when the regions of interest (e.g. lung area) are not properly extracted before the next steps of segmentation and nodule detection.

Edge-based CT segmentation techniques have been also commonly used for lung nodule detection. In (Campadelli et al., 2006), first derivative of Gaussian filters were computed along four cardinal directions and then an edge tracking method based on the Laplacian of Gaussian (LoG) operator was used in order to detect the boundaries of lung nodules. Authors in (Mendonça et al., 2004) automatically selected rectangular areas as the ROIs. The edge points were then detected using spatial edge detector filters and combined to determine a closed contour of the edges and boundaries. In (Korfiatis et al., 2014), two-dimensional wavelet transform of the images was used to determine the boundaries of the lung. Authors in (Mansoor et al., 2014) adapted the fuzzy connectedness image segmentation to develop a generic lung parenchyma extraction approach. In (Kishore and Satyanarayana, 2013), a comprehensive evaluation of several edge detectors techniques and their efficiency in lung nodule detection applications in noisy environments was carried out.

Using the edge-based segmentation techniques is often challenging in the noisy circumstances. To be more clear, if the lung CT data is infected with noise, finding the edges and then extracting the nodules may be not accurate. In such cases, some unreal edges and boundaries may be produced. On the other hand, since the edges extracted by the edge-based segmentation and the real object boundaries are not always the same, these methods cannot guarantee an appropriate segmentation. Therefore, edge detection is often considered only as a preprocessing step, not a segmentation procedure as a whole. Some other state-of-the-art methods dealing with nodule detection using various types of segmentation and classification techniques can be found in (Setio et al., 2016; Nagarajan et al., 2013; Valente et al., 2016; Bergtholdt et al., 2016; Zhou et al., 2015; Zhou et al., 2016).

The main goal of our study is to present a lung nodule detection method that can handle the abovementioned challenges, while still follows a relatively straight-forward algorithm. The presented article proposes an automatic approach in which both the threshold-based and edge-based segmentation methodologies are applied together. In the proposed method, a combination of mathematical morphology operations is used in order to detect the edges and extract the regions of interest. Using statistical region merging algorithm, the segments are created for each layer of data and consequently, the 3D objects are produced for the whole multi-layer CT data. The 3D objects (as the nodule candidates) are then analyzed and assigned to be nodule or non-nodule structures using a supervised classifier. In this article, the support vector machine classifier is used for classifying the extracted 3D objects into nodule and non-nodule groups. SVM is a supervised learning algorithm firstly proposed in 1992 and mainly used for classification and regression 
applications (Boser et al., 1992). Various kernels can be exploited when running the SVM classifiers. In this paper, the Radical Basis Function (RBF) kernel is used for implementation of SVM. The parameters of this kernel are optimized using a five-fold cross validation. Moreover, we propose to extract six spectral/ morphological/textural features from all candidates for the classification step. These features will be discussed in section two in more details. In order to enhance the gained results and to make the proposed approach more robust against noises and other types of irregularities, a diffusion-based approach which exploits the nonlinear Partial Differential Equations (NLPDE) is also used as a pre-processing step. Applying the nonlinear version of the diffusion-based spatial smoothing step leads to well-smoothed images which their edges are kept unchanged.

In the presented approach, there are two types of parameters: the adaptively tuned parameters, and the fixed parameters. The values of the former parameters (e.g. the NLPDE parameters) are adaptively set with regard to the spatial/spectral characteristics of the CT data. The values of the latter type of parameters (e.g. the main parameter of the SRM algorithm) are predefined as fixed values. Therefore, there is no need to manually tune the parameters during the nodule detection procedure. This explains our reason to call the proposed method a 'fully-automatic' nodule detection approach. The briefed diagram of the proposed algorithm is shown in Fig. 1.

The rest of the paper is as follows. The proposed methodology is presented in section two as a combination of four sub-sections: NLPDE-based pre-processing, morphological-based ROI extraction, SRMbased segmentation, and nodule detection based on SVM classification. The quantitative outcomes of the experimental implementations are reported in section three. The fourth section includes discussions on the gained results while conclusions and future works are drawn in section five.

\section{MATERIALS AND METHODS}

\section{NONLINEAR PARTIAL DIFFERENTIAL EQUATIONS BASED SMOOTHING}

CT images are generally noise-free images, however, they may occasionally contain some unwanted irregularities. Smoothing the image increases the homogeneity of the extracted objects and removes probable redundant noises and artifacts. This leads to reasonably smaller number of objects which are often more meaningful. Therefore, as the first step of the proposed pulmonary nodule detection framework, we propose applying a pre-processing level in which each layer of the input data is smoothed.

The smoothing technique used in this article is based on the nonlinear partial differential equations. The main reason of choosing NLPDE rather than the other traditional smoothing/denoising methods (such as mean, median, Gaussian, and Weiner filtering, wavelet-based methods, etc.) is its capability in presserving the edges and boundaries which is very vital in image segmentation and object detection applications (Perona and Malik, 1990).

Scale-space diffusion filtering and more specifically nonlinear PDE-based smoothing/denoising techniques have been introduced in (Perona and Malik, 1990; Witkin and Filtering, 1984), respectively. PDE-based approaches smooth the images while retaining their main structures and boundaries unchanged. Non-linear diffusion equation is defined as below (Zhou et al., 2016):

$$
\frac{\partial I(x, y, r)}{\partial t}=\nabla \cdot(c(x, y, r) \nabla I(x, y, r)
$$

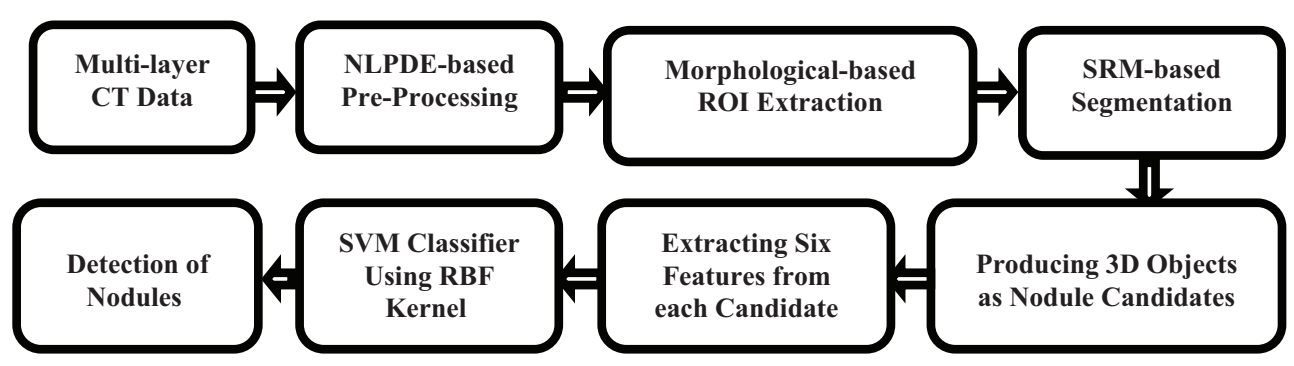

Fig. 1. The briefed block diagram of the proposed Approach. 
Where $\nabla$ and $\nabla$. are gradient and divergence operators, respectively. Moreover, $l(x, y, r)$ is the smoothed image after $r^{\text {th }}$ round of applying the smoothing algorithm. In addition, $c(x, y, r)$ denotes the diffusion coefficient which itself is a nonlinear function of the image gradients (in nonlinear versions of PDEs) around each pixel (Perona and Malik, 1990; Zehtabian et al., 2015). In the present article, the following nonlinear relation between the diffusion coefficient and the image gradients (i.e., $\nabla \mathrm{I}$ ) has been used, as suggested in (Zehtabian et al., 2015):

$$
c(x, y, r)=\frac{1}{\left(1+\frac{|\nabla I|^{2}}{k^{2}}\right)} .
$$

In the above equation, $k$ is an important parameter which is used to control the diffusion power and con-sequently the smoothing level. Higher values of this parameter lead to more powerful smoothing. On the other hand, very low values of this parameter result in images which are not adequately smoothed or denoised.

As suggested in (Perona and Malik, 1990), in order to transform Eq. 1 to its iterative discrete form, the following equation is used in which $d_{N}, d_{S}, d_{E}, d_{W}$ are derivatives along the north, south, east and west directions, respectively, and $\Delta t$ is the time step which is fixed to 0.25 in the present study:

$$
\begin{gathered}
I(x, y, t+\Delta t)=I(x, y, t)+ \\
+\Delta t\left(d_{N} \cdot c_{N}+d_{S} \cdot c_{S}+d_{E} \cdot c_{E}+d_{W} \cdot c_{W}\right) .
\end{gathered}
$$

Using Eq. 2, the diffusion coefficients can be calculated along the four directions and then inserted in Eq. 3. The discrete numerical solution shown in Eq. 3 must be iterated several times to guarantee a wellsmoothed image.

The number of iterations (i.e., $N$ ) in Eq. 3 as well as parameter $k$ in Eq. 2 can be tuned manually or automatically. Authors in (Zehtabian et al., 2015) proposed a mathematical approach to adaptively set this parameter for restoration of the noisy remote sensing data. To this end, they first calculated the gradients around all pixels in the image along all eight cardinal and diagonal directions. This resulted in eight matrices which were depicted by $d_{n}, d_{s}, \ldots$, $d_{n e}, d_{n w}, \ldots$, etc. As an example, $d_{n e}$ was the gradient matrix along the north-eastern direction.

The differences between the eight gradient matrices are then computed in horizontal, vertical and diagonal directions. Two examples are shown in below:

$$
\begin{gathered}
d_{n s}=d_{n}-d_{s} \\
d_{n w s e}=d_{n w}-d_{s e}
\end{gathered}
$$

Each element of the four resulted matrices was then powered by two. They used notations $d_{n s}^{\mathscr{\Theta}}, d_{e w}^{\mathscr{Q}}, d_{n e s w}^{\mathscr{Q}}, d_{n w}^{\mathscr{\Theta}}$ se (for the new matrices. Eq. 5 and Eq. 6 were then used for achieving two new matrices, among which first was related to the gradient differences along cardinal directions and latter was related to the diagonal directions.

$$
\begin{gathered}
\varphi_{c}=\sqrt[2]{d_{n s}^{(2)}+d_{e w}^{(2)}}, \\
\varphi_{d}=\sqrt[2]{d_{n e s w}^{(2)}+d_{n w s e}^{(2)}} .
\end{gathered}
$$

After calculating the variances of elements in matrices $\varphi_{c}$ and $\varphi_{d}$ using Eq. 7 and Eq. 8, the parameter $F$ which shows the average value of the results, can be driven as Eq. 9 .

$$
\begin{aligned}
& v_{c}=\operatorname{var}\left(\varphi_{c}\right), \\
& v_{d}=\operatorname{var}\left(\varphi_{d}\right), \\
& F=\frac{\left[v_{c}+v_{d}\right]}{2} .
\end{aligned}
$$

As shown in Eq. 10, parameter $k$ can be adaptively calculated by multiplying $F$ by a fixed scale factor:

$$
K_{\text {adapt }}=F \times \gamma .
$$

Supported by extensive sets of experiments, the authors in (Zehtabian et al., 2015) proved a linear relation between parameter $k$ and the value of $F$. They also suggested to set parameter $N$ (refer to Eq. 3 ) with regard to the value of $F$. In the present article, we use a similar technique in order to automatically find the proper values of $k$ and $N$ for various CT images with different textural and spectral characterristics. However, since there are some differences between our application (i.e., image smoothing) and the goal of what proposed in (Zehtabian et al., 2015) (i.e., image restoration), we need a lower level of diffusion-based process. Therefore, the value of the scaling factor $\gamma$ used in our study is lower than what the authors used in (Zehtabian et al., 2015). Fig. 2 shows the results of applying the NLPDE-based preprocessing algorithm to a given $\mathrm{CT}$ image of lung. In this experiment, the value of parameters $k$ and $N$ are automatically set to 24 and 30, respectively. As can be seen in Fig. 2, the smoothing step smoothed the image and removed the unwanted artifacts, while preserving the edges and the other important details. 


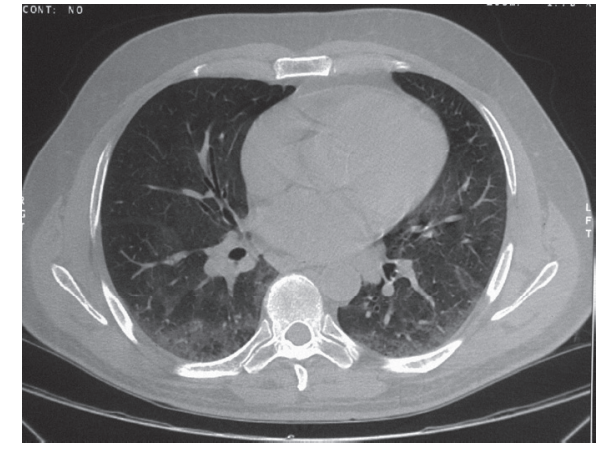

CT Image before PDE Smoothing

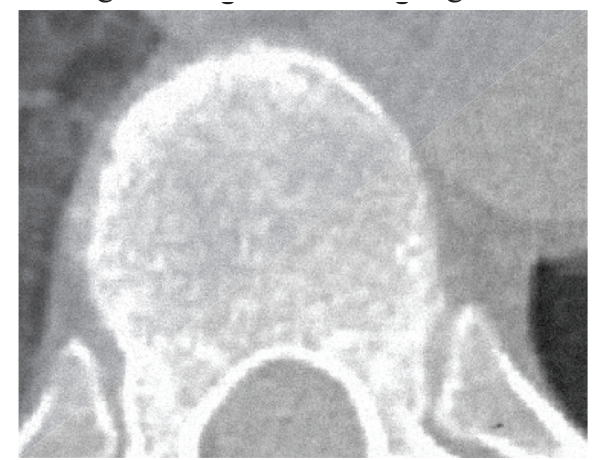

Cropped Area of the initial CT Image

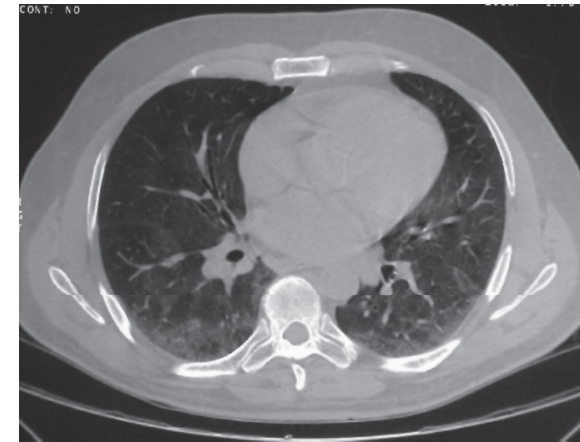

CT Image after PDE Smoothing

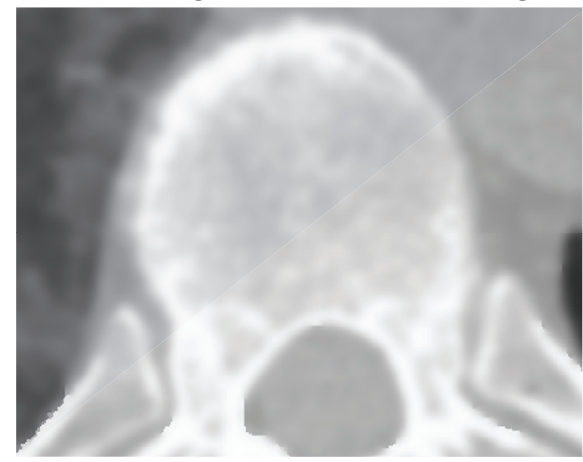

Cropped Area of the Smoothed Image

Fig. 2. The result of applying the automatic nonlinear PDE-based smoothing step to a given CT image. For better assessment, two cropped areas from the initial and smoothed images are shown as well.

\section{EXTRACTION OF THE REGIONS OF INTEREST (ROIS)}

After smoothing all layers of the input CT data, the regions of interest (ROIs) should be extracted from each layer of the data, individually. Since the goal of our research is to detect and evaluate the lung nodules, we expect this step to provide at least two main objects which represent the lungs. Those pixels in each layer of the CT data which are located in these regions will be then analyzed in the next steps, while the other areas in the images will be exempt from further processing. By removing the redundant areas, the ROI extraction step usually results in reducing the computational time needed for processing the next steps of the proposed method. It also often increases the accuracy of the nodule detection algorithm.

In the present article, we propose a ROI extraction algorithm which is mainly based on morphological filtering. Mathematical morphology filters have been commonly exploited in image processing (Soille, 2013; Crespo et al., 1995; Zehtabian and Ghassemian, 2016a). Four widely-used morphological operators comprise erosion, dilation, opening and closing. In the erosion process, the brighter objects in image will shrink, while the objects which are already darker than their neighboring area will expand (Soille, 2013). On the contrary, the dilation makes the brighter objects larger while decreasing the size of the darker objects (Soille, 2013). The opening operator is made of an ero-sion operator followed by a dilation operator, while the closing is the composition of dilation followed by erosion. All the four above-mentioned operations utilize structuring elements (SE) in their calculations. The size and shape of SE define which objects in an image will be eliminated after applying the morphological operators and which objects will remain. As an example, after applying the dilation operator to a given region, the dark objects which are smaller than SE (or cannot contain it because of their shape) will be eliminated while the larger objects will remain mostly intact. Because of the importance of edges in CT images, we have used the geodesic versions of the morphological operators which are also called "morphological reconstruction" (Crespo et al., 1995; Zehtabian and Ghassemian, 2016a). These operators are beneficial especially where preserving the edges and shapes of objects in images is an important goal. Such goal seems more important in CT images in which the objects are often small and closely located. Fig. 3 illustrates the block-diagram of the ROI extraction algorithm used in the proposed nodule detection framework. In order to assess the effect of applying each block of this block-diagram to a given layer of a CT data, the step-by-step visual results are also shown in this figure. 


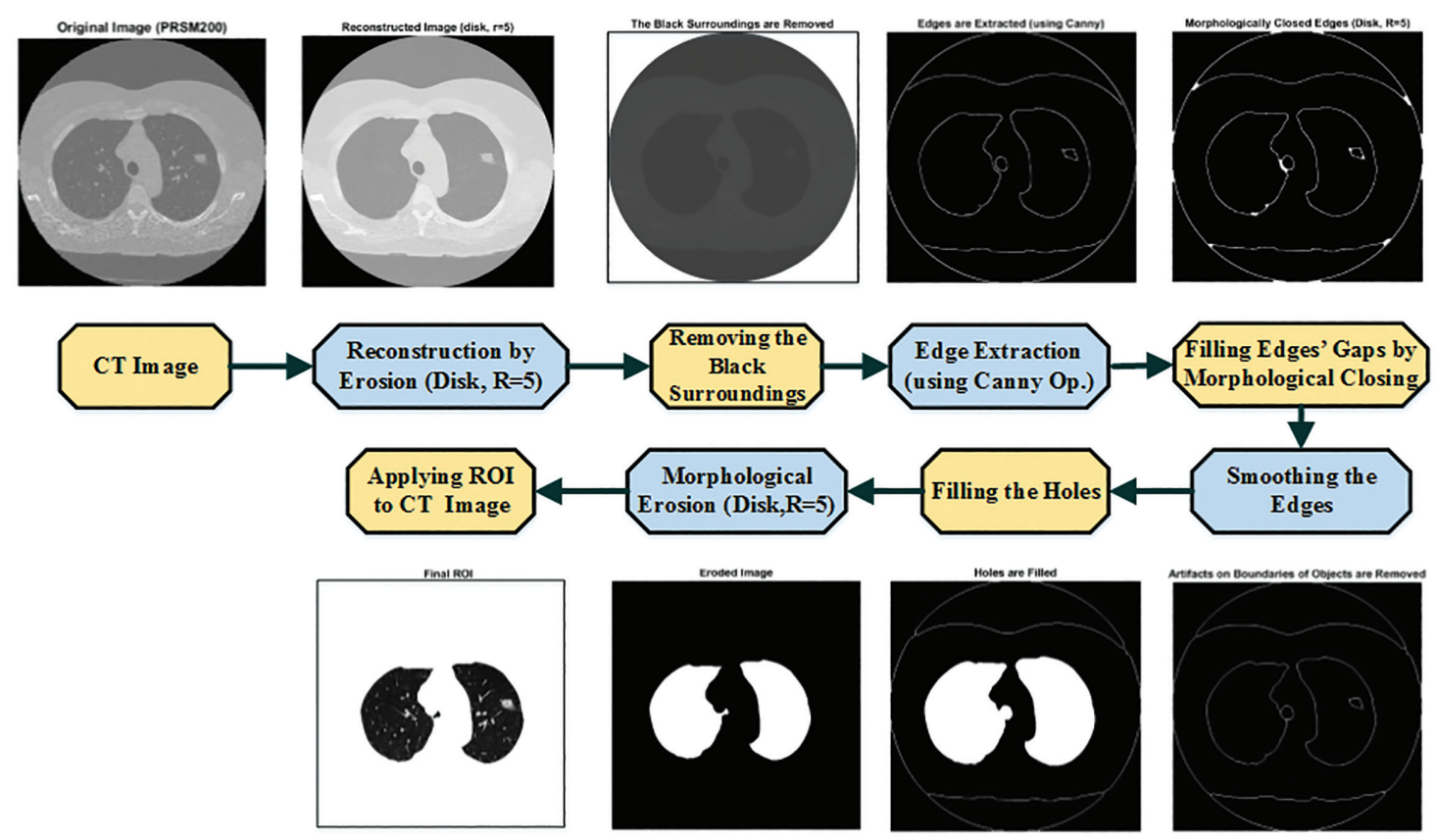

Fig. 3. Block-diagram of the ROI extraction algorithm used in the proposed nodule detection framework.

As illustrated in Fig. 3, the input CT image is first fed into a morphological reconstruction block. In this step, using a disk-shaped structural element (SE), first an erosion operator is applied to the image. The morphological reconstruction is then performed using the eroded image as the mask and the input image as the marker. The resulted image is more suitable for edge extraction since some textures which do not contain strong edges are faded or entirely removed.

In order to remove the black surroundings and limit our focus to the central circle-shaped area of the CT image, the gray-level of those pixels with the minimum intensity are substituted with the maximum possible gray-level (e.g., '32767' for a 16-bits CT data). The edges of the image are then extracted using the Canny edge detection operator which follows a multistep algorithm in order to identify different types of boundaries in the pictures.

After applying the morphological closing operation (which utilizes a disk-shaped SE which its radius is equal to 5), some unwanted gaps in the edges are then filled. As stated in the previous paragraphs, the morphological closing operation is a dilation followed by an erosion. In order to smooth the edges, artifacts on the boundaries of objects are removed using morpho-logical filtering in such a way that the objects are not allowed to break apart. The remained pixels make up the image skeleton. In Fig. 4, we have distinguished the extracted objects of a given CT data previously shown in Fig. 3, each with a different color.

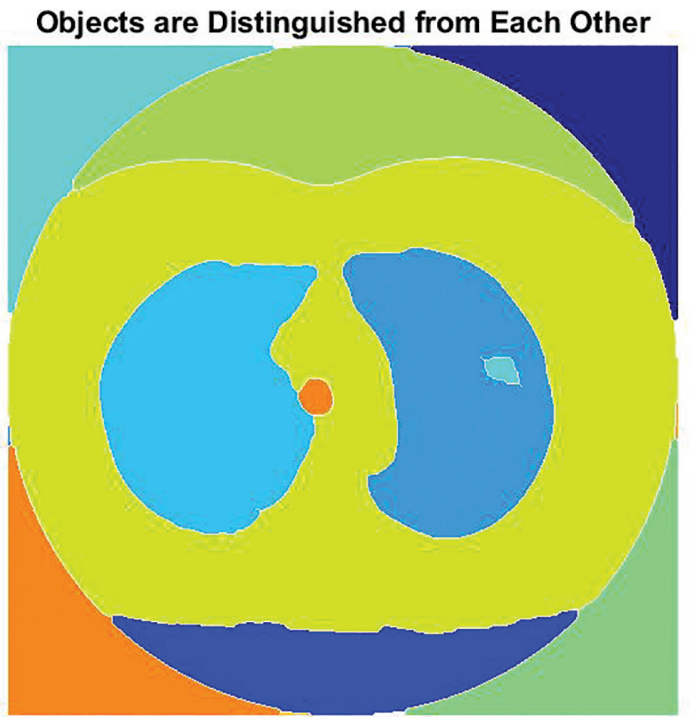

Fig. 4. Objects extracted by the proposed morphological based algorithm. In order to clearly show the separated areas, they are colored with different colors.

In the next step, the holes in the binary image are filled. In our application, a hole is a set of background pixels that cannot be reached by filling in the background from the edges of the image. After this step, the two main areas which contain the lungs are distinguished from the other areas. The image is then fed into a morphological erosion block where its unwanted edges are removed. The resulted mask is finally applied to the initial image and the regions of interest are ready to be processed by the next stages of the proposed nodule detection framework. 


\section{STATISTICAL REGION MERGING (SRM) BASED SEGMENTATION}

SRM is a widely used method in image processing which converts an image palette with greater number of colors into an image palette with fewer colors. In this mapping, same colors are merged together (Nock and Nielsen, 2004; Zehtabian and Ghassemian, 2016b). SRM is often considered as a fast and accurate algorithm for image segmentation (Nock and Nielsen, 2004). A SRM-based segmentation algorithm comprises both region growing and merging procedures. The algorithm assesses pixels within an area and merges them into a connected group of pixels based on an integration criterion. It is similar to creating a group of new generations within a population based on a certain criteria.

SRM is a region growing and merging technique, since it first checks if two neighbouring pixels belong to the same segment and then merges the pixels if pre-defined criteria is satisfied (Nock and Nielsen, 2004). To this end, a set of neighbouring pixel pairs are prepared. Using a sort function, the couples of pixels are then sorted in an ascending order. The sort function is given in Eq. 11, where $p$ and $p$ ' are the intensities of a given pixel and its neighbour, respectively (Nock and Nielsen, 2004):

$$
\mathrm{f}\left(p, p^{\prime}\right)=\left|p-p^{\prime}\right| .
$$

After sorting the pixel pairs, the SRM segmentation criterion is used in order to merge the neighboring pixels. To this end, all sorted pairs are consecutively fed into the segmentation criterion shown in Equation (12). At the first step of the SRM segmentation algorithm, those pairs which are able to satisfy the criterion are merged together. The next steps of the SRM segmentation algorithm deal with merging the neighboring segments rather than the pixels.

$$
\begin{aligned}
\mathrm{P}\left(\mathrm{R}, \mathrm{R}^{\prime}\right)= & \begin{cases}\text { True: } & \text { if } \sqrt{\sum_{b}\left|\bar{R}_{b}-\bar{R}_{b}^{\prime}\right|^{2}} \leq \\
\text { False: } & \text { otherwise }\end{cases} \\
& \leq \operatorname{sqrt}\left(N_{b}+1\right) \times \sqrt{H^{2}(\mathrm{R})+H^{2}\left(\mathrm{R}^{\prime}\right)},
\end{aligned}
$$

in which:

$$
H(R)=g \sqrt{\left(\frac{1}{2 Q|R|} \ln \left(\frac{\left|R_{|R|}\right|}{\delta}\right)\right)},
$$

where $\mathrm{g}$ is the maximum number of grey-levels in the input image (e.g., 256), $R$ is a given segment, $\bar{R}$ is the mean value of the grey-levels in $R$, and $\delta=1 / 6 \times I^{2}$. Moreover, parameter $Q$ determines the amount of roughness and smoothness of segmentation. With higher values of $Q$, more segments are produced and vice versa (Nock and Nielsen, 2004).

Fig. 5 shows the effect of varying the value of segmentation parameter $Q$ on the segmentation maps resulted by the SRM algorithm. In this figure, in order to facilitate the comparison of the maps obtained by different parameters, each segmentation map is colorized in a shuffled manner in which adjacent segments are randomly colored.

As can be seen in this figure, increasing the value of the segmentation parameter leads to an increment in the number of segments and vice versa. As an example, for $Q=30$, only a few number of segments are produced. When $Q$ reaches 50, some newer segments are produced as well. For very large values of $Q$, the number of segments is very high in such a way that for extremely large $Q$, the number of extracted segments reach the number of all pixels which are located in the regions of interest. In order to achieve desired results and avoid over-segmentation and undersegmentation problems, the value of the parameter $Q$ must be properly set. In the present study, this value is manually tuned after extensive sets of experiments. Therefore, we suggest setting this para-meter to a fixed value (i.e., $Q=100$ ) for all the lung CT datasets.

After applying the SRM segmentation algorithm to each layer of the multilayer CT data, several segmentation maps are produced each containing a probably different (but often close) number of extracted objects. These objects are then separated from their surrounding background. As a visual example, Fig. 6 illustrates the segment maps resulted by applying the SRM algorithm to four continuous layers (i.e., $195^{\text {th }}$ to $198^{\text {th }}$ ) of a lung CT data named 'CaseSM0052' from ELCAP dataset. In this figure, extracted ROIs are also shown for each layer of the data. As can be inferred from Fig. 6, the extracted objects of the adjacent layers are located in relatively similar places, thus they can form three-dimensional (3D) volumes if they connect together.

In order to detect the nodules, the two-dimensional objects from adjacent layers must be first converted to three-dimensional objects which are considered as nodule candidates. To this end, we propose to connect the boundaries of each extracted object from each layer to those of an object from adjacent layers, only if a criterion is met. To be more clear, if object $O_{a}$ from layer $b_{i}$ intersects with object $O_{b}$ in the adjacent layer $b_{i+1}$ in at least five pixels, all pixels placed on the boundary of the former object are connected to their corresponding pixels on the boundary of the latter object. This procedure results in several three-dimensional objects which must be then categorized into nodules and non-nodules groups. 


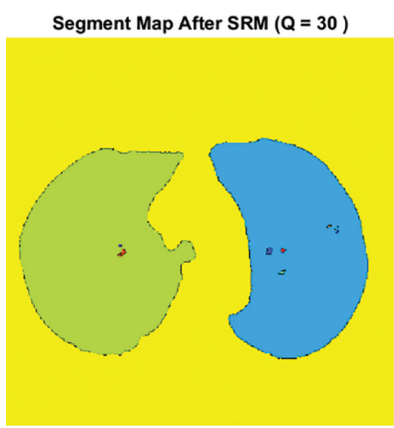

$\mathbf{Q}=\mathbf{3 0}$

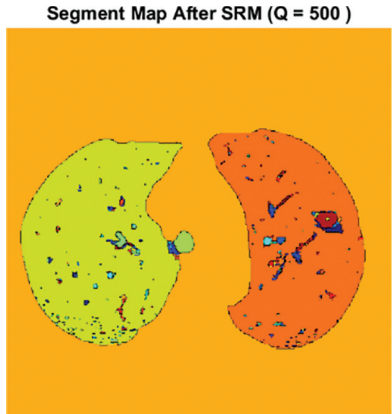

$\mathbf{Q}=\mathbf{5 0 0}$

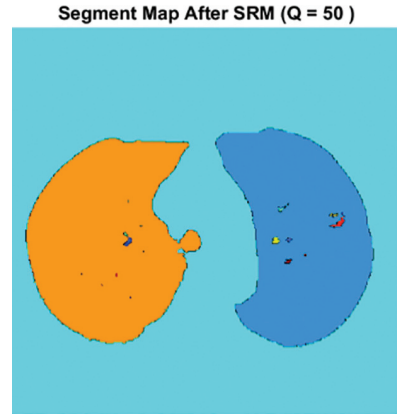

$\mathbf{Q}=\mathbf{5 0}$

Segment Map After SRM $(Q=1000)$

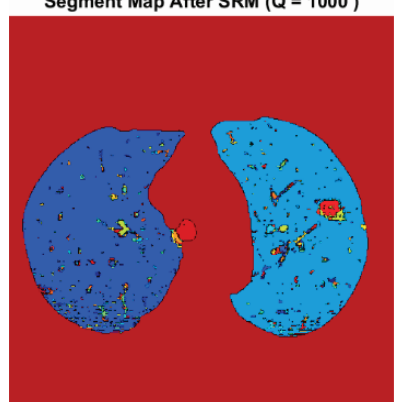

$\mathbf{Q}=\mathbf{1 0 0 0}$

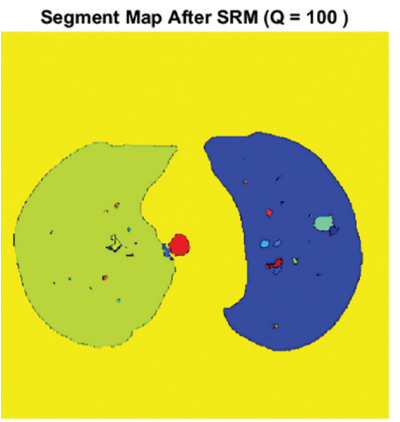

$Q=100$

Segment Map After SRM $(Q=5000)$

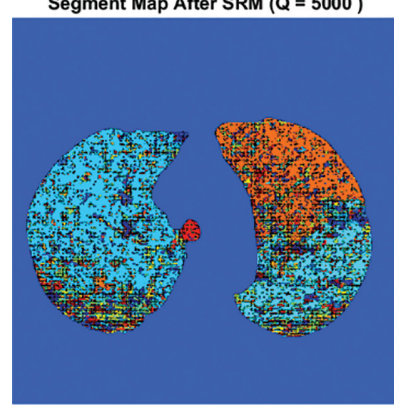

$\mathbf{Q}=\mathbf{5 0 0 0}$

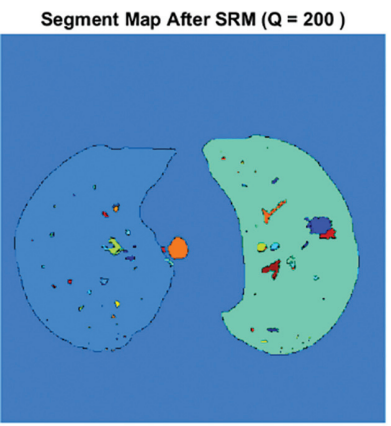

$Q=200$

Segment Map After SRM $(Q=10000)$

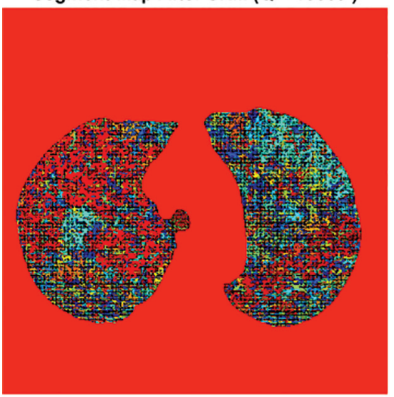

$Q=10000$

Fig. 5. Effect of varying the value of parameter $Q$ on the segmentation maps produced by the SRM algorithm. Each map is colorized in a shuffled manner.

CaseSM0052 $\cdot$ Layer $=195$

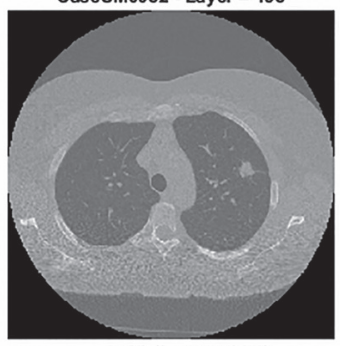

ROI (Layer 195)

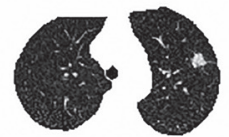

SRM Map - Layer $195-(Q=100)$

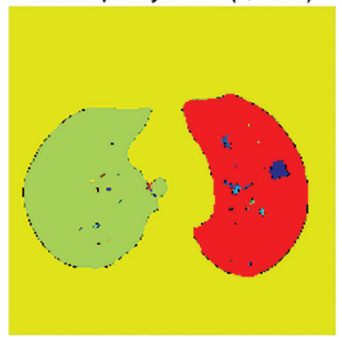

CaseSM0052 - Layer $=196$

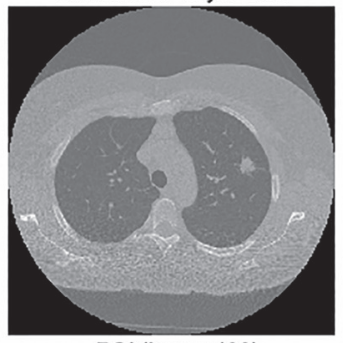

ROI (Layer 196)

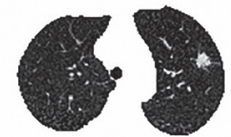

SRM Map - Layer 196 - $(Q=100)$

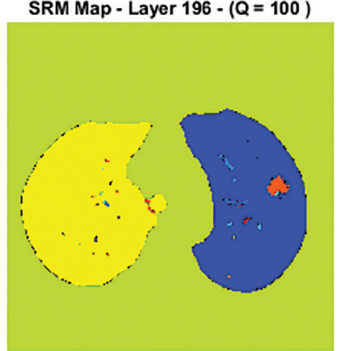

CaseSM0052 - Layer $=197$

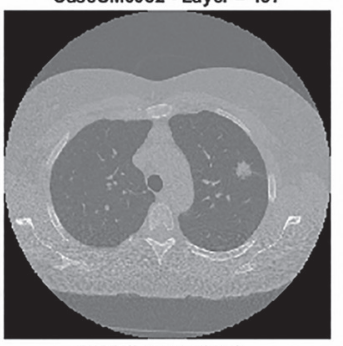

ROI (Layer 197)

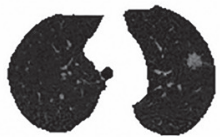

SRM Map - Layer 197 - $(Q=100)$

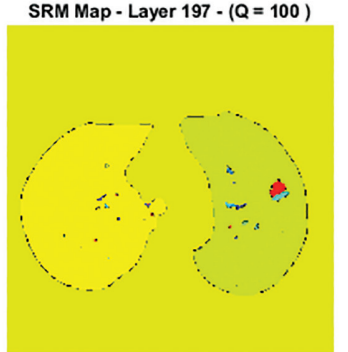

CaseSM0052 - Layer $=198$

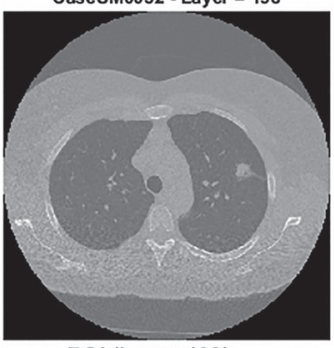

ROI (Layer 198)

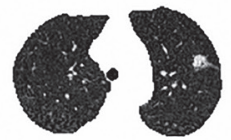

SRM Map - Layer $198-(Q=100)$

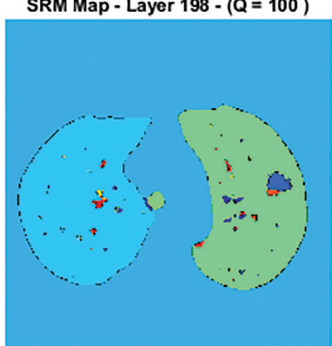

Fig. 6. Visual results of applying the SRM segmentation algorithm to four continuous layers of a lung CT data (Case: SM0052). Upper row: gray-level illustrations of the $195^{\text {th }}, 196^{\text {th }}, 197^{\text {th }}$, and $198^{\text {th }}$ layers of the data; Middle row: extracted ROIs corresponding to the layers; Lower row: segment maps corresponding to the layers, while $Q=100$. 


\section{SVM-BASED CLASSIFICATION}

After extracting the nodule candidates, the true nodules should be distinguished from the non-nodule objects. The non-nodule structures mainly belong to natural small tissues in the lungs or be caused by redundant details or artifacts which have not yet removed by the previous pre-processing step. In order to distinguish the nodules from the other non-nodule structures, we suggest applying a support vector machine classifier which has been previously trained using adequate number of training data, comprising the true nodules and non-nodules. To this end, six features are first calculated from each nodule candidate. These features are then analyzed using the SVM classification which had been previously trained by the training nodules and non-nodules. If the features of a given $3 \mathrm{D}$ object fit the predefined requirements of the true nodules, the object is supposed to be a nodule, and vice versa.

The six features utilized in our classification step comprise four spectral, one morphological, and one textural features. The four spectral features are directly determined according to the gray level of the pixels located within each nodule candidate. These are the mean, minimum, maximum and the standard deviation of the gray levels of pixels which are located inside the nodule candidates. Such spectral features have been chosen due to this fact that the lung nodules often have higher gray levels compared to the other natural tissues such as vessels (Boroczky et al., 2006).

Since the fifth used feature considers the shape of the nodule candidates, it is regarded as a morphological feature. This feature characterizes the compactness of the $3 \mathrm{D}$ objects that is defined as the ratio between the volume equivalent radius and spatial deviation equivalent radius, as introduced in (Boroczky et al., 2006).

Finally, a textural feature is also utilized in the classification step which is extracted using the rose diagram technique. The rose diagram is a feature extraction technique which is utilized for extracting the angular-directional information of the 3D objects (Froz et al., 2017). To this end, some directional measurements such as local gradients are often exploited. In the present article, the Sobel filter is used in order to calculate the local gradients and consequently to extract the angular-directional features such as the mean direction of the gradient angles, the circular variance, and the circular standard deviation. Further information about the above-mentioned features as well as some other alternative textural features can be found in (Boroczky et al., 2006; Froz et al., 2017; Padma and Giridharan, 2016; Harikumar, 2015; Devan et al., 2014).

\section{RESULTS}

In this section, experimental results of applying the proposed nodule detection approach are reported. Several 3D CT images taken from two widely-used databases have been used in our experiments. The first database (usually named as ELCAP public lung dataset) has been produced by the Cornell University (http://www.via.cornell.edu/lungdb.html, Accessed May 2016). It contains $50 \mathrm{CT}$ images of the chest which are commonly used to evaluate the performance of the computer-aided detection systems (http://www. via.cornell.edu/lungdb.html, Accessed May 2016). Each of these low-dose CT images consists of about 260 slices and comprises single or multiple nodules. The spatial resolution of the images are mainly about $0.7 \times 0.7 \times 1.25 \mathrm{~mm}$.

The second used database is a freely-available set of CT images and is called as 'LIDC-IRDI' (which stands for 'Lung Image Database Consortium and the Image Database Resource Initiative') (Armato et al., 2011). The nodule characteristics are also available for this dataset, especially for nodules which their diameters are larger than $3 \mathrm{~mm}$ or smaller than 30 $\mathrm{mm}$ (Froz et al., 2017). In the experiments carried out in the present article, $800 \mathrm{CT}$ images (out of total 1012 images) of this dataset have been used.

Although the locations of nodules are predetermined by the radiologists and are already provided in the used databases, in order to obtain a more fair comparison between the different methods, the gained results have been evaluated by two clinical experts (who are qualified enough to manually detect the nodules in lung CT images), as well. However, since the scores achieved by the two evaluation scenarios (i.e., referring to the predetermined nodule maps, as well as using the manual evaluation by the two experts) show a considerably high correlation, we only report the results of the former evaluation scenario.

In order to evaluate the performance of the proposed approach and the competing methods, first the lung CT images in each dataset are divided into two groups: the train group and the test group. Several criteria are then used for assessing the gained results. These criteria comprise the detection sensitivity, accuracy, and specificity (as suggested in (Armato et al., 2011; Fielding and Bell, 1997; Mousa and Khan, 2002). These criteria are functions of some simpler metrics such as True Positive (TP), False Positive (FP), False Negative (FN) and True Negative (TN) (Lo et al., 1995). The TP (TN) is the percentage of true nodules (true non-nodules) which have been correctly classi- 
fied as nodules (non-nodules). Moreover, the FP (FN) is the percentage of true non-nodules (true nodules) which have been incorrectly classified as nodules (non-nodules).

On the other hand, the detection sensitivity is the ratio between $\mathrm{TP}$ and the sum of $\mathrm{TP}$ and $\mathrm{FN}$. The detection accuracy is the ratio between $(\mathrm{TP}+\mathrm{TN})$ and $(\mathrm{TP}+\mathrm{TN}+\mathrm{FP}+\mathrm{FN})$, while the detection specificity is the ratio between $\mathrm{TN}$ and the sum of TN and FP (Armato et al., 2011; Fielding and Bell, 1997; Mousa and Khan, 2002).

Since the SVM classifier needs to be trained, each database is divided into two groups: training and testing. In this paper, $20 \%$ of images in each database are randomly selected for training the classifier, while the remaining $80 \%$ are used for the testing step. Moreover, in order to ensure a fair evaluation of the proposed method, the abovementioned selection is permuted three times for the LIDC-IRDI case and the obtained results are averaged and reported.

Quantitative assessments of the results gained by the proposed approach show a considerable perfor- mance. As some examples, the detection accuracy of the proposed method is about $90.0 \%$ when applied to fifty CT images of the ELCAP dataset and is near to 95.1\% while applied to $800 \mathrm{CT}$ images of the LIDCIRDI dataset. Along with the proposed method, Table 1 contains the results of applying nine other nodule detection methods to similar datasets. The competing methods are based on Pixon-based segmentation (Hassanpour et al., 2011), template matching and neural classifier (Hasanabadi et al., 2014), hybrid features (Akram et al., 2016), Level-Set method (Silveira et al., 2007), a method based on genetic algorithm (GA) and SRM (Zehtabian and Ghassemian, 2016b), context curve calculation (Zhang et al., 2013), circular features based method (Mousa and Khan, 2002), a method based on threshold clustering and GA (de Carvalho et al., 2014), and a method based on artificial crawlers feature extraction and SVM (Froz et al., 2017). Among the competing methods, the first five methods have been implemented again by the authors of the present article on the new dataset(s), but under similar circumstances and conditions. The results of the other four methods have been taken from the literature.

Table 1. Comparison results of applying the proposed method as well as other competing methods to three datasets (i.e., the ELCAP dataset, LIDC-IRDI dataset, and a proprietary dataset). Best results are bolded in terms of each criterion.

\begin{tabular}{|c|c|c|c|c|c|}
\hline Nodule Detection Methods & Database & $\begin{array}{c}\text { Number of } \\
\text { used CT } \\
\text { Images }\end{array}$ & $\begin{array}{c}\text { Sensitivity } \\
\mathbf{\%}\end{array}$ & $\begin{array}{c}\text { Specificity } \\
\mathbf{\%}\end{array}$ & $\begin{array}{c}\text { Accuracy } \\
\mathbf{\%}\end{array}$ \\
\hline Proposed Method & ELCAP & 50 & $92.5 \%$ & $87.5 \%$ & $90.0 \%$ \\
\cline { 2 - 5 } & LIDC-IRDI & 800 & $\mathbf{9 2 . 4 \%}$ & $95.3 \%$ & $95.1 \%$ \\
\hline $\begin{array}{c}\text { Template Matching \& Neural Classifier } \\
\text { (Hasanabadi } \text { et al., 2014) }\end{array}$ & LIDC-IRDI & 800 & $82.1 \%$ & $85.6 \%$ & $84.5 \%$ \\
\hline $\begin{array}{c}\text { Hybrid Features Based Method } \\
\text { (Akram } \text { et al., 2016) }\end{array}$ & LIDC-IRDI & 800 & $88.7 \%$ & $91.3 \%$ & $90.1 \%$ \\
\hline $\begin{array}{c}\text { Level-Set Method } \\
\text { (Silveira } \text { et al., 2007) }\end{array}$ & LIDC-IRDI & 800 & $84.3 \%$ & $85.9 \%$ & $84.8 \%$ \\
\hline $\begin{array}{c}\text { GA-SRM Method } \\
\text { (Zehtabian and Ghassemian, 2016b) }\end{array}$ & LIDC-IRDI & 800 & $89.7 \%$ & $92.7 \%$ & $92.3 \%$ \\
\hline $\begin{array}{c}\text { Pixon-based Method } \\
\text { (Hassanpour } \text { et al., 2011) }\end{array}$ & LIDC-IRDI & 800 & $78.8 \%$ & $81.2 \%$ & $80.4 \%$ \\
\cline { 2 - 6 } & ELCAP & 50 & $72.5 \%$ & $60 \%$ & $67.5 \%$ \\
\hline $\begin{array}{c}\text { Context Curve Calculation } \\
\text { (Zhang } \text { et al., 2013) }\end{array}$ & ELCAP & 50 & $\mathrm{NA}$ & $\mathrm{NA}$ & $82.5 \%$ \\
\hline $\begin{array}{c}\text { Circular Features Based Method } \\
\text { (Mousa and Khan, 2002) }\end{array}$ & Non-Standard & 50 & $87.5 \%$ & $62.5 \%$ & $75 \%$ \\
\hline $\begin{array}{c}\text { Threshold Clustering and GA } \\
(\text { de Carvalho } \text { et al., 2014) }\end{array}$ & LIDC-IRDI & 833 & $85.9 \%$ & $\mathbf{9 7 . 7 \%}$ & $\mathbf{9 7 . 6 \%}$ \\
\hline $\begin{array}{c}\text { Crawlers Feature Extraction and SVM } \\
\text { (Froz } \text { et al., 2017) }\end{array}$ & LIDC-IRDI & 833 & $91.9 \%$ & $94.8 \%$ & $94.3 \%$ \\
\hline
\end{tabular}


Fig. 7 shows visual result of applying the abovementioned procedure to three different $\mathrm{CT}$ images from the ELCAP dataset. The first row of Fig.7 shows example layers of the three images. In each subfigure of the second row of Fig.7, the volumes of all 3D objects are calculated and then plotted as a curve for each case. In the third row, the 3D representtation of one detected nodule for each case is shown. In order to create better visualizations of the extracted nodules, we have used an interpolation technique while connecting the borders of the objects together.

\section{DISCUSSION}

As can be inferred from Table 1, the proposed approach outperforms the other competing methods except the method which is based on threshold clustering and genetic algorithm (de Carvalho et al., 2014). The me- thod based on the crawlers features and SVM (Froz et al., 2017) is the third ranked as well. The average detection accuracy of our approach is about $90.0 \%$ for ELCAP dataset and about $95.1 \%$ for LIDC-IRDI dataset. For the methods proposed in (de Carvalho et al., 2014) and (Froz et al., 2017), this ratio is $97.6 \%$ and $94.3 \%$ for LIDC-IRDI case, respectively. However, the proposed method excels the others in terms of detection sensitivity. Among the competing methods, the traditional Pixon-based approach (Hassanpour et al., 2011) shows the least detection accuracy. Although the Pixon-based segmentation technique has been later improved in (Zehtabian and Ghassemian, 2016a) by the same authors, since the main concern of their modified methodology (i.e., classification of the multispectral remote sensing data) was totally different from ours, the advanced version of their method is exempt from being considered in our comparison table.
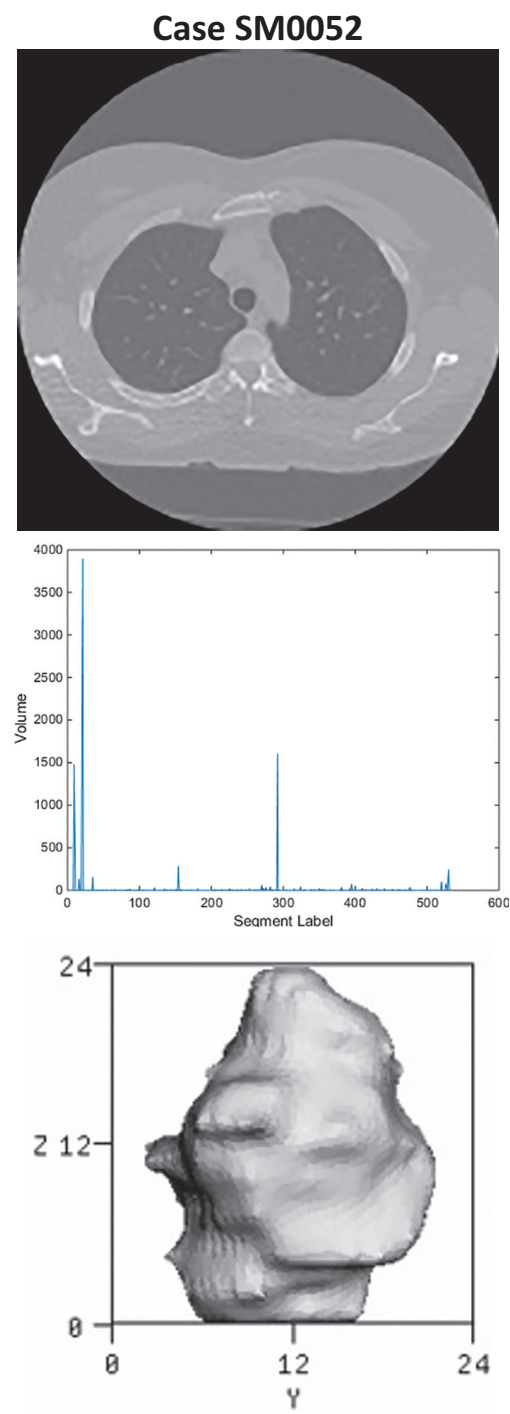
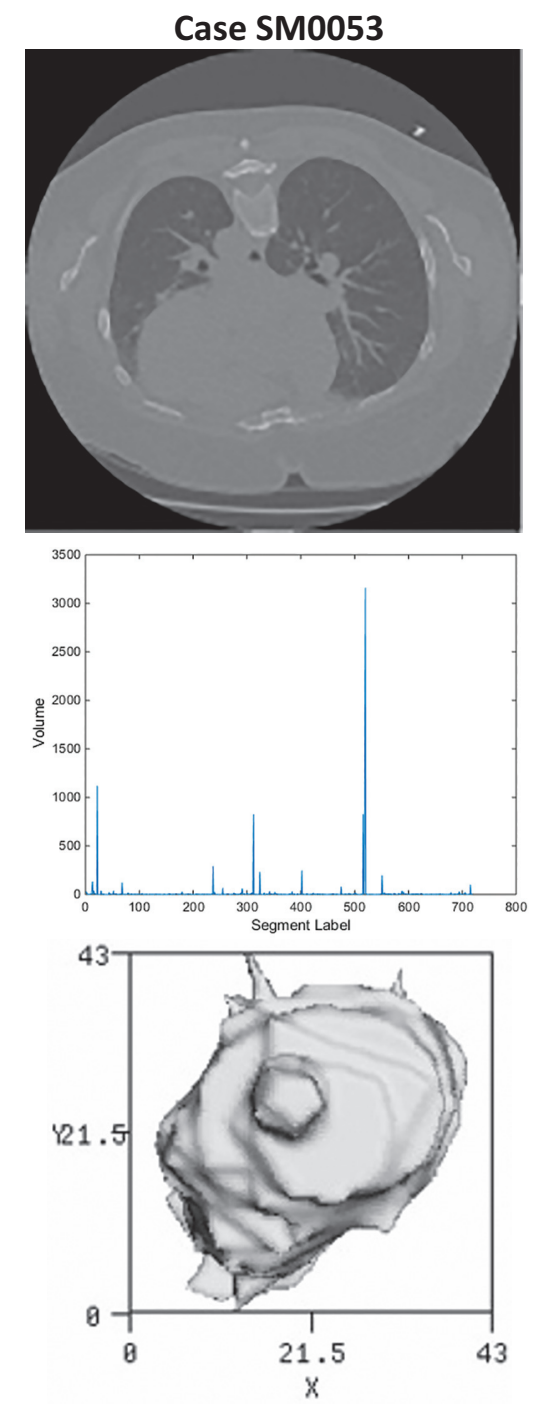
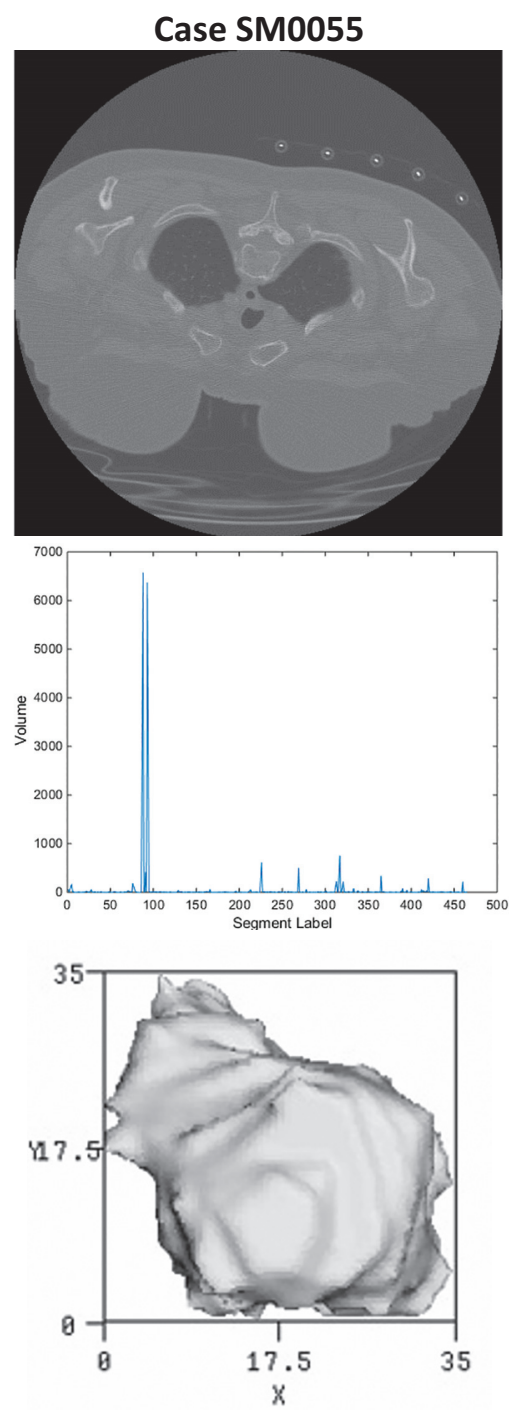

Fig. 7. Visual result of applying the proposed to three CT images from the ELCAP dataset taken from the lung area. 
As the only competing method which utilizes the SRM technique, the results of the GA-SRM method in (Zehtabian and Ghassemian, 2016b) are reported in Table 1, as well. However, it must be noted that the method in (Zehtabian and Ghassemian, 2016b) is not inherently an object detection method. To be clearer, this method only acts as a segmentation technique. Therefore, in order to convert the GA-SRM method (Zehtabian and Ghassemian, 2016b) to a nodule detection approach, we have combined this method with our proposed nodule detection procedure. In other words, each layer of the CT image is first segmented using a single-layer version of the GA-SRM method and then we apply the 3D object creation step as well as the feature extraction and SVM classification steps to the segment maps. As can be seen in Table 1, even using the same feature extraction and classification steps, the proposed approach outperforms the competing GA-SRM method. It is mainly because of the following reasons: the proposed approach makes benefit from an innovative ROI extraction technique as well as the PDE-based preprocessing step in which the parameters are optimized.

On the other hand, the efficiency of the proposed method in noisy environments is considerable. To be more clear, exploiting the PDE-based preprocessing step results in significant reduction of the effects of additive noises and artifacts on the nodule detection performance. From the computational point of view, the proposed approach possesses the second rank after the Pixon-based method (Hassanpour et al., 2011), while most the competing methods are implemented in Matlab2015 utilizing an Intel CPU with $3.20 \mathrm{GHz}$ and $4 \mathrm{~GB}$ of ram. Using the mentioned hardware, the proposed approach averagely needs about 12 seconds to perform all the three levels (i.e., preprocessing, ROI-extraction, and the final segmentation) to each layer of the CT image. The 3D object creation and the final classification also take less than two seconds to run. As the fastest technique among the competing methodologies, the Pixon-based schema (Hassanpour et al., 2011) only needs about 8 seconds to segment each layer. On the other hand, the method proposed in (Zehtabian and Ghassemian, 2016b) is the most time consuming one among the competing methods since it averagely needs about 85 seconds for segmenting each layer of the $\mathrm{CT}$ data. This relatively high computational time is not surprising since the GAPDE based technique makes benefit from the genetic algorithm which itself is naturally time consuming (Zehtabian and Ghassemian, 2016b).

\section{CONCLUSION AND FUTURE WORKS}

The present article proposes a new methodology for automatic detection of the lung nodules using an innovative combination of PDE, SRM, morphological filtering, and 3D object extraction steps. In the proposed method, first a PDE diffusion based preprocessing step (in which the main parameter is adaptively set) is used for smoothing the data as well as removing the noise and artifacts. Using several levels of morphological processing, the regions of interest are extracted from the image and then fed into the statistical region merging algorithm. After this step, a unique segmentation map is produced for each layer of the multilayer CT data. The 2D segments are then transformed into 3D objects. Six features from each of these objects are extracted and fed into a SVM classifier in order to distinguish the true nodules from the non-nodules.

For the evaluation of the approaches, they are applied to several sets of data containing both real lung CT images as well as two standard image datasets titled 'ELCAP public lung dataset' and 'LIDC-IRDI'. In the case of the standard datasets, the efficiencies of the nodule detection methods have been assessed referring to the reference nodule maps that are available for each data. Meanwhile, two clinical experts have also validated the results obtained after applying the nodule detection methods to the real and standard lung images.

Along with considerable accuracy, specificity, and sensitivity of our method in detection of the pulmonary nodules, its relatively straight-forward framework and the fully-automatic procedure that it offers are two other main contributions of the proposed PDESRM-based technique. Among the two groups of parameters used in the presented approach, the first group (such as parameter ' $k$ ' in PDE which is tuned according to the spatial/spectral characteristics of the CT images) is adaptively determined. The second group of parameters (such as the SRM parameter) are predefined after consulting with clinical experts and then uniquely applied to all the $\mathrm{CT}$ images. As a result, there is no need to manually tune the parameters during the presented algorithm. In our future works, however, we will mainly focus on optimization and adaptation of all the vital parameters used in various steps of the framework, especially the main parameter of SRM (i.e., $Q$ in Eq. 13). It will probably increase the robustness of the proposed nodule detection ap- 
proach specially when faced with irregular environmental conditions or other types of multimodal images.

\section{REFERENCES}

Akram S, Javed MY, Akram MU, Qamar U, Hassan A (2016). Pulmonary Nodules Detection and Classification Using Hybrid Features from Computerized Tomographic Images. J Med Imag Health Info 6:252-9.

Alpert JB, Lowry CM, Ko JP (2015). Imaging the solitary pulmonary nodule. Clini Chest Med 36:161-78.

Armato III SG, McLennan G, Bidaut L, McNitt-Gray MF, Meyer CR, Reeves AP, Kazerooni EA (2011). The lung image database consortium (LIDC) and image database resource initiative (IDRI): a completed reference database of lung nodules on CT scans. Med Phy 38:915-31.

Bergtholdt M, Wiemker R, Klinder T (2016). Pulmonary nodule detection using a cascaded SVM classifier. In SPIE Medical Imaging. Int Soc Opt Phot 978513.

Boroczky L, Zhao L, Lee KP (2006). Feature subset selection for improving the performance of false positive reduction in lung nodule CAD. IEEE Trans Info Tech Biomed, 10:504-11.

Boser BE, Guyon IM, Vapnik VN (1992). A training algorithm for optimal margin classifiers. In Proceed fifth annual workshop Comp learning theory, 144-52.

Campadelli P, Casiraghi E, Artioli D (2006). A fully automated method for lung nodule detection from posteroanterior chest radiographs. IEEE trans Med Imag 25: 1588-603.

Crespo J, Serra J, Schafer RW (1995). Theoretical aspects of morphological filters by reconstruction. Sig Process 47:201-25.

De Carvalho Filho AO, de Sampaio WB, Silva AC, de Paiva AC, Nunes RA, Gattass M (2014). Automatic detection of solitary lung nodules using quality threshold clustering, genetic algorithm and diversity index. Art Intel Med 60:165-77.

Devan L, Santosham R, Hariharan R (2014). Automated texture-based characterization of fibrosis and carcinoma using low-dose lung CT images. Int J Imag Sys Tech 24:39-44.

El-Baz A, Beache GM, Gimel'farb G, Suzuki K, Okada K, Elnakib A, Abdollahi B (2013). Computer-aided diagnosis systems for lung cancer: challenges and methodologies. Int J Biomed Imag.

Fielding AH, Bell JF (1997). A review of methods for the assessment of prediction errors in conservation presence/ absence models. Environ Conserv 24:38-49.

Froz BR, de Carvalho Filho AO, Silva AC, de Paiva AC, Nunes RA, Gattass M (2017). Lung nodule classifycation using artificial crawlers, directional texture and support vector machine. Exp Sys App 69:176-88.
Gao Q, Wang S, Zhao D, Liu J (2007). Accurate lung segmentation for X-ray CT images. In Third Int Conf Natural Comp (IEEE - ICNC 2007):275-9.

Gould MK, Fletcher J, Iannettoni MD, Lynch WR, Midthun DE, Naidich DP, Ost DE (2007). Evaluation of patients with pulmonary nodules: when is it lung cancer?: ACCP evidence-based clinical practice guidelines. Chest J 132:108-30.

Harikumar R (2015). Performance analysis of neural networks for classification of medical images with wavelets as a feature extractor. Int J Imag Sys Tech 25:33-40.

Hasanabadi H, Zabihi M, Mirsharif Q (2014). Detection of pulmonary nodules in CT images using template matching and neural classifier. J Adv Comp Research 5:19-28.

Hassanpour H, Zehtabian A, Yousefian H (2011). Pixonbased image segmentation. INTECH Open Access Publisher.

Herman GT (2009). Fundamentals of computerized tomography: image reconstruction from projections. Springer Science \& Business Media.

Hoffman EA, Clough AV, Christensen GE, Lin CL, McLennan G, Reinhardt JM, Wang G (2004). The comprehensive imaging-based analysis of the lung: A forum for team science1. Acad Radio 11:1370-80.

Hu S, Hoffman EA, Reinhardt JM (2001). Automatic lung segmentation for accurate quantitation of volumetric X-ray CT images. IEEE Trans Med Imag 20:490-8.

John J, Mini MG (2016). Multilevel Thresholding Based Segmentation and Feature Extraction for Pulmonary Nodule Detection. Pro Tech 24:957-63.

Kim DY, Kim JH, Noh SM, Park JW (2003). Pulmonary nodule detection using chest $\mathrm{CT}$ images. Acta Radio 44:252-7.

Kishore VV, Satyanarayana RVS (2013). Performance evaluation of edge detectors-morphology based ROI segmentation and nodule detection from DICOM lung images in the noisy environment. In Adv Comp Conf (IEEE- IACC), 1131-7.

Korfiatis P, Skiadopoulos S, Sakellaropoulos P, Kalogeropoulou C, Costaridou L (2014). Combining 2D wavelet edge highlighting and 3D thresholding for lung segmentation in thin-slice CT. British J Radio.

Lo SC, Lou SL, Lin JS, Freedman MT, Chien MV, Mun SK (1995). Artificial convolution neural network techniques and applications for lung nodule detection. IEEE Trans Med Imag 14:711-8.

Mansoor A, Bagci U, Xu Z, Foster B, Olivier KN, Elinoff JM, Mollura DJ (2014). A generic approach to pathological lung segmentation. IEEE Trans Med Imag 33: 2293-310.

Mendonça AM, da Silva JA, Campilho A (2004). Automatic delimitation of lung fields on chest radiographs. In Biomedical Imaging: Nano to Macro, 2004. IEEE Int Symp, 1287-90. 
Mousa WA, Khan MA (2002). Lung nodule classification utilizing support vector machines. In Imag Process Proceedings. Int Conf, 150-3.

Nagarajan MB, Huber MB, Schlossbauer T, Leinsinger G, Krol A, Wismüller A (2013). Classification of small lesions in breast MRI: evaluating the role of dynamically extracted texture features through feature selection. J Med Bio Eng 33:102-10.

Nock R, Nielsen F (2004). Statistical region merging. IEEE Trans Patt Analy Machine Intell 26:1452-8.

Padma A, Giridharan N (2016). Performance comparison of texture feature analysis methods using PNN classifier for segmentation and classification of brain CT images. Int J Imag Sys Tech 26:97-105.

Penedo MG, Carreira MJ, Mosquera A, Cabello D (1998). Computer-aided diagnosis: a neural-network-based approach to lung nodule detection. IEEE Trans Med Imag 17:872-80.

Perona P, Malik J (1990). Scale-space and edge detection using anisotropic diffusion. IEEE Trans Patt Analy Machine Intell 12:629-39.

Setio AAA, Ciompi F, Litjens G, Gerke P, Jacobs C, van Riel SJ, et al. (2016). Pulmonary nodule detection in ct images: false positive reduction using multi-view convolutional networks. IEEE Trans Med Imag 35: 1160-9.

Silveira M, Nascimento J, Marques J (2007). Automatic segmentation of the lungs using robust level sets. In 2007 29th Annual Int Conf IEEE Eng Med Bio Soc, 4414-7.

Soille P (2013). Morphological image analysis: principles and applications. Springer Science \& Business Media.

Valente IRS, Cortez PC, Neto EC, Soares JM, de Albuquerque VHC, Tavares JMR (2016). Automatic 3D pulmonary nodule detection in CT images: a survey. Comp Meth Prog Biomed 124:91-107.
Wei J, Li G (2014). Automated Lung Segmentation and Image Quality Assessment for Clinical 3-D/4-D-Computed Tomography. IEEE J Trans Eng Health Med 2: $1-10$.

Witkin AP, Filtering SS (1984). A new approach to multiscale description. In IEEE ICASSP.

Yim Y, Hong H, Shin YG (2005). Hybrid lung segmentation in chest CT images for computer-aided diagnosis. In Proceedings of 7 th Int Workshop Enterprise Net Comp Healthcare Indust, 2005. HEALTHCOM 2005, 378-83.

Zehtabian A, Nazari A, Ghassemian H, Gribaudo M (2015). Adaptive Restoration of Multispectral Datasets used for SVM classification. Europ J Remote Sens 48:183-200.

Zehtabian A, Ghassemian H (2016). Automatic ObjectBased Hyperspectral Image Classification Using Complex Diffusions and a New Distance Metric. IEEE Trans Geosc Remote Sens 54:4106-14.

Zehtabian A, Ghassemian H (2016). Combining Genetic Algorithm with PDEs for Improving the Performance of Statistical Region Merging Based Object Extraction. J Ind Soc Remote Sens, 1-13.

Zhang F, Song Y, Cai W, Zhou Y, Shan S, Feng D (2013). Context curves for classification of lung nodule images. In Digital Imag Comp: Tech App (DICTA), 2013 Int Conf, 1-7.

Zhou Z, Wu S, Chang KJ, Chen WR, Chen YS, Kuo WH, Tsui PH (2015). Classification of benign and malignnant breast tumors in ultrasound images with posterior acoustic shadowing using half-contour features. J Med Bio Eng 35:178-87.

Zhou T, Lu H, Zhang, J, Shi H (2016). Pulmonary Nodule Detection Model Based on SVM and CT Image FeatureLevel Fusion with Rough Sets. BioMed Research Int, 2016. 